\title{
Pilot experiments for the International Thermonuclear Experimental Reactor active beam spectroscopy diagnostic
}

\author{
M. von Hellermann, ${ }^{a)}$ M. de Bock, R. Jaspers, and K. Jakubowska \\ Nieuwegein, NL \\ R. Barnsley, C. Giroud, N. C. Hawkes, and K. D. Zastrow \\ UKAEA Culham Laboratory Euratom Association, Abingdon, United Kingdom \\ P. Lotte and R. Giannella \\ CEA Cadarache, Association Euratom, France
}

FOM-Institute for Plasma physics "Rijnhuizen," Association EURATOM, Trilateral Euregio Cluster, 3430 BE

A. Malaquias

IAEA, Vienna, Austria

E. Rachlew

Euratom Association-VR, KTH Stockholm, Sweden

S. Tugarinov and A. Krasilnikov

TRINITI Troitsk, Russia

\author{
A. Litnovsky, V. Philipps, and P. Wienhold \\ Institute for Plasma Physics, FZ-Juelich, Euratom Association, 52425 Juelich, Germany \\ P. Oelhafen and G. De Temmerman \\ Institut für Physik, University of Basel, 4056 Basel, Switzerland \\ L. Shmaenok \\ Phystex, Vaals, NL and loffe Physical Technical Institute, St. Peterburg, Russia
}

(Presented on 19 April 2004; published 1 October 2004)

Supporting pilot experiments and activities which are currently considered or already performed for the development of the International Thermonuclear Experiment Reactor active beam spectroscopy diagnostic are addressed in this article. Four key issues are presented including optimization of spectral instrumentation, feasibility of a motional Stark effect (MSE) evaluation based on line ratios, "first-mirror" test-bed experiments at the tokamak TEXTOR, and finally the role of integrated data analysis for the conceptual layout of the change exchange recombination spectroscopy and MSE diagnostic. (C) 2004 American Institute of Physics. [DOI: 10.1063/1.1787950]

\section{INTRODUCTION}

A comprehensive package of active beam based spectroscopy tools for the International Thermonuclear Experimental Reactor (ITER) has been developed and a final summary was recently completed. ${ }^{1}$ The package encompasses charge exchange recombination spectroscopy $(\mathrm{CXRS})^{2,3}$ for the measurement of the main impurity ion densities (including helium ash), ion temperature and torodial as well as poloidal plasma rotation. Quantitative use of beam emission spectroscopy (BES) ${ }^{4}$ based on comprehensive atomic modeling is proposed as an indispensable cross-calibration tool for absolute local impurity density measurements ${ }^{5}$ and monitoring of the neutral beam power deposition profile. Finally, a full exploitation of the motional Stark effect (MSE) pattern is proposed to deduce local pitch angles, total magnetic fields ${ }^{6,7}$ and radial electric fields. CXRS on slowing-down alphas as demonstrated. on the Tokamak Fusion Test Reactor ${ }^{8}$ is also

\footnotetext{
${ }^{\text {a) }}$ Author to whom correspondence should be addressed; electronic mail: mgvh@rijnh.nl
}

considered for the ITER DNB and HNB (100 keV/amu, 2.2 MW diagnostic neutral beam and $500 \mathrm{keV} / \mathrm{amu}, 17 \mathrm{MW}$ heating neutral beam respectively) and was discussed recently. ${ }^{2}$ More quantitative studies and modeling will be required in this field. A similar diagnostic challenge refers to the measurement of slowing-down beam ions as produced by the HNB. Medium energy velocity distribution functions representing slowing-down helium beam ions were observed at the Joint European Tours (JET). ${ }^{9}$ A number of issues have been recognized over the last years which need to be addressed in advance of the next stage, the diagnostic "procurement phase." Four of those are addressed in this article.

(1) The optimization of spectral instruments and detectors for the active beam spectroscopy diagnostic package needs to be tested on existing fusion devices in order to establish suitable combinations for high-resolution and broadband applications. Moreover, multi-tasking recommends practical maintainable instrumental solutions.

(2) A converging interest in motional Stark diagnostics, beam emission spectroscopy, and charge exchange spec- 


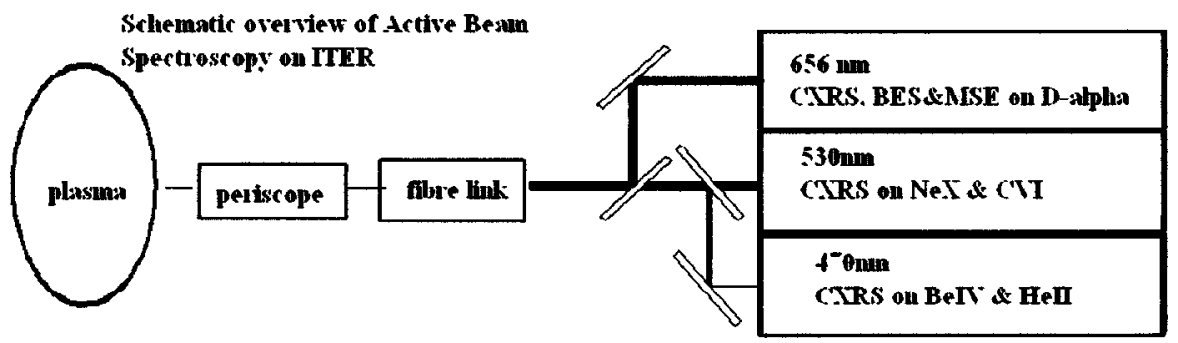

FIG. 1. Schematic layout of CXRS/ BES/MSE spectroscopic instrumentation. Multi-tasking of periscopes for simultaneous evaluation of CXRS (impurity and bulk ions), BES on injected neutrals and finally for MSE ratiometry.

troscopy has highlighted the needs for characterization of the polarization transfer properties of optical periscopes.

(3) A central issue is the survival and protection of the first mirrors used in observation periscopes. The effects of carbon and beryllium coatings on reflectivity and polarization characteristics of metallic mirrors have been modeled and presented at several meetings (e.g., Ref. 7). First results describing erosion effects of monocrystalline molybdenum mirrors were reported recently. ${ }^{10}$

(4) Finally, data evaluation procedures are closely linked to feasibility studies and will ultimately decide on achievable performances.

\section{CXRS AND BES INSTRUMENTATION}

Very early on it has been recognized that the substantial attenuation of the diagnostic beam requires high-optical throughput spectrometers in order to compensate for a low CX signal in the presence of huge background of continuum radiation. For the case of a modulated DNB the effective signal to noise is then determined by the ratio of $\mathrm{CX}$ signal and fluctuation of the background. A crucial criterion for the acceptance of CXRS as a viable tool for ITER is the capability of measuring local helium ash densities in the plasma core. At TRINITI a high-optical-throughput, high-resolution spectrometer was developed especially for CXRS application. ${ }^{11}$ A unique combination of a large $f$-number $(F / 3), f=480 \mathrm{~mm}$, and high linear dispersion value $(D$ $=0.25-0.3 \mathrm{~nm} / \mathrm{mm})$ make it a particularly suited instrument for the envisaged ITER CXRS system. The system's etendue $\left(A \cdot \Omega=4 \times 10^{-1} \mathrm{~mm}^{2} \mathrm{sr}\right)$ is preserved by the periscope fiber bundle assembly, and a DNB slab of about $100 \times 100 \mathrm{~mm}$ is imaged for each radial channel onto the entrance slit (1 $\times 5 \mathrm{~mm}$ ) of individual spectrometers. The role of the CXRS/ BES diagnostic as a multi-tasking requires the simultaneous measurement of the main ion species: helium ash at $468 \mathrm{~nm}$, intrinsic impurities $\mathrm{C}$ and $\mathrm{Ne}$ at $529 \mathrm{~nm}$, and bulk D and $\mathrm{T}$ ions at $650 \mathrm{~nm}$ (cf. Fig. 1). The proposed scheme is to make use of color filters and different orders of the TRINITI echelle grating instruments for different representative wavelengths. Pilot experiments are required addressing achievable sensitivities, stray-light suppression, and color control. A critical issue will also be the spectral purity of the underlying continuum radiation and its quantum noise photon statistics in order to verify predicted spectral signal-to-noise reactor (SNR) values (cf Ref. 1). The detection of nonthermal broadband features depends critically on clear distinction of CX induced features and passive background.

\section{MSE MEASUREMENTS BY RATIOMETRY}

The combination of CXRS and beam emission spectroscopy ${ }^{5}$ is seen today as the sole path to obtain absolute ion densities (helium ash) on ITER. BES refers here explicitly to a quantitative exploitation of measured and modeled line intensities and wavelength separations and not to the measurement of plasma fluctuations. A natural spin-off of BES on the DNB is to exploit the D-alpha spectrum and its amplitudes for magnetic diagnosis. This will be complementary to MSE polarimetry on the HNB. ${ }^{6,12,13}$ In fact, the complementary exploitation of the entire MSE polarization pattern will ultimately optimize signal accuracy and spatial resolution. Whereas the viewing geometry of the HNB MSE periscopes is optimized for a maximum sensitivity to a rotation of the polarization pattern, the DNB periscopes will only be sensitive to changes of the angle between Lorentz-field vector and viewing line. For the ITER magnetic field $B_{t}$ $=5.2 \mathrm{~T}$, and a DNB energy of $100 \mathrm{keV} / \mathrm{amu}$, the MSE components ( $6 \pi$ and $3 \sigma$ components, respectively) will be well separated $(>5 \AA)$ and the SNR compared to the background fluctuation level (assuming photon statistics of continuum radiation) should be in excess of 50 (see also Refs. 1 and 2).

The pitch angle $B_{p} / B_{t}$ derived from the dipole intensity ratio of $\sigma$ and $\pi$ group in MSE spectrum, which, in the case of statistical population, is determined by the angle $\vartheta$ between direction of 1.o.s. and Lorentz-vector $E_{L}=v \times B$

$$
\frac{I_{\pi}}{I_{\sigma}}=\frac{\sin ^{2} \vartheta}{1+\cos ^{2} \vartheta} .
$$

Maximum sensitivity for observation angles $\vartheta$ close to $45^{\circ}$. For the ITER CXRS top-port periscope the angle $\vartheta$ on axis $\left(B_{p}=0\right)$ is $32.6^{\circ}$. Figure 2 shows $q$ values derived from ratio $I_{\pi} / I_{\sigma}$ for the case of a simplified circular magnetic flux surface and the ITER CXRS top-port periscope. In contrast to polarimetry, no active or passive polarizing element is involved in ratiometry. However, a key issue will be isotropic reflection factors ensuring the preservation of $I_{\pi} / I_{\sigma}$ for the entire observation system including multiple mirrors in the periscope, the fiber link, and finally in the spectrometer itself. A MSE ratiometry pilot experiment is presently implemented on TEXTOR in ITER-like geometry.

\section{FIRST-MIRROR EXPERIMENTS ON TEXTOR}

Metallic mirrors which are foreseen for ITER optical periscopes were recently investigated on TEXTOR. The aim of the experiments was to investigate optical properties of plasma-facing mirrors in dependence on erosion, deposition, and particle implantation. 


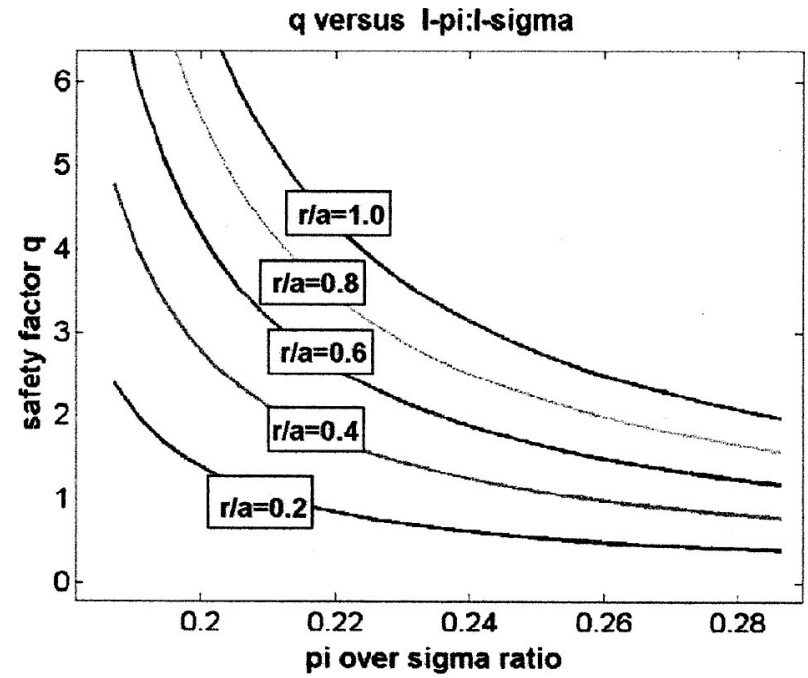

FIG. 2. Safety factor $q$ as derived from MSE intensity ratio for different minor radii and ITER top-portconfiguration. Note, a simplified case off circular flux geometry has been assumed for illustration purposes.

Large polycrystalline metal mirrors (molybdenum and tungsten) on inclined target holders were exposed in the scrape-off layer (SOL) plasma of TEXTOR in both erosion and deposition-dominated zones. The accumulated plasma exposure time of mirrors was about $900 \mathrm{~s}$. Particle energies and particle affluence were of the same order of magnitude as those expected for ITER. Figure 3 shows a schematic layout of the mirror test and one sample exposed in the deposition-dominated zone. The reflectivity was measured before and after exposure in the SOL. The wavelength regime of measurements ranged from 250 to $2500 \mathrm{~nm}$. First experimental evaluations ${ }^{14}$ can be summarized as:

(1) after plasma exposure the reflectivity was decreased by up to $35 \%$ in the deposition areas. The reflectivity was increased by $12 \%$ in the plasma near zones (erosion zones);

(2) fringes due to constructive and destructive interference are observed if $\lambda / 4$ corresponds to the optical thickness of a deposited film;

(3) the degradation of reflectivity of the exposed mirror

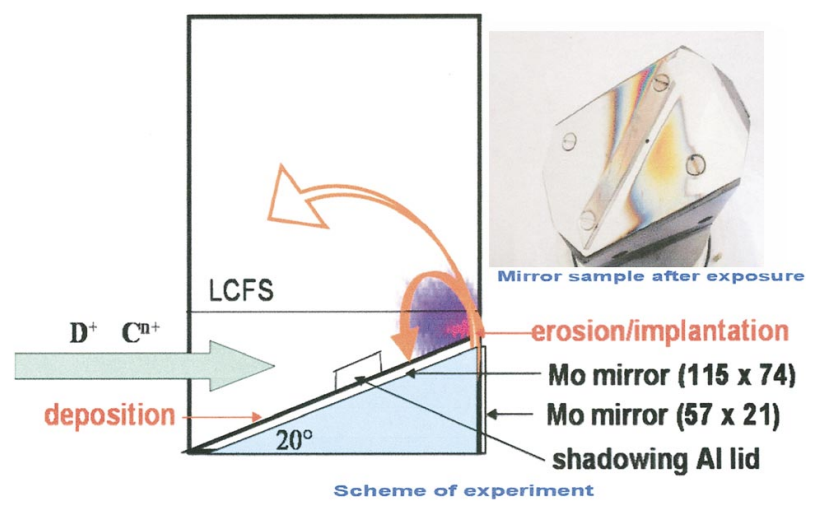

FIG. 3. (Color) Large polycrystalline metal mirrors (molybdenum and tungsten) on inclined target holders were exposed in the SOL plasma of TEXTOR in both erosion and deposition-dominated zones. samples is likely due to film growth observed on the mirror surface; and

(4) the increase of reflectivity of mirrors noticed on some areas is possibly due to annealing effects during the temperature excursions and/or cleaning by plasma ions.

\section{ACTIVE BEAM SPECTROSCOPY DATA ANALYSIS: CHEAP FOR ITER}

A collateral activity to ongoing conceptual design and feasibility studies is dedicated to the development of suitable data analysis packages including spectral analysis and physics evaluation. At JET the self-consistent CX evaluation package CHEAP (charge exchange analysis package) is used routinely and is potentially applicable in inter-shot performance.

The CHEAP concept is based on a comprehensive atomic modeling ${ }^{15}$ of all emission processes (beam emission, active and passive charge exchange recombination) making use of a full set of data for the plasma environment. An essential first step is the introduction of a common mapping grid for all physical parameters. This strategy has enabled us to treat the beam-plasma interaction (i.e., beam stopping and beam emission processes) in a consistent fashion. Multiple step processes and contributions from excited states are taken into account. For ITER the concept of integrated data CX analysis implies a comprehensive diagnostic coverage of the main plasma ions (intrinsic and seeded impurities and bulk ions). From this point of view, the issue of CX measurement of helium ash densities on ITER is inseparable from a simultaneous measurement of all ions. Moreover, the issue of a density measurement is linked directly to a measurement of ion temperature and plasma rotation. below:

Highlights of future activities in this field are listed

(1) Super Fit: The present strategy of spectral analysis is usually based on a step-by-step process addressing each spectrum individually. A new concept is to involve a full set of spectra representing, for example, a complete ion temperature profile and solve all spectra in one go.

(2) Integrated Data Analysis: From a similar point of view, a spectral analysis is ultimately to be seen as part of a global data consistency strategy. Ion temperature and density lead to ion pressure and combined with electron pressure to a match of kinetic and diamagnetic energy which can be used as a constraint on spectral analysis.

(3) The stochastic occurrence of plasma edge line emission, e.g., edge localized modes ELMs is a potential hazard to a perfect background suppression scheme using beam modulation. In this case, complex spectra will have to be evaluated requiring advanced atomic modeling and suitable extraction tools.

\footnotetext{
${ }^{1}$ M. von Hellermann, "Active Charge Exchange Spectroscopy (CXRS) and Beam Emission Spectroscopy (BES+MSE) with Diagnostic Neutral Beam (DNB)" (under EFDA contract; not publicly available, contact: mgvh@rijnh.nl)

${ }^{2}$ M. von Hellermann, C. Giroud, N. C. Hawkes, R. Jaspers, A. Krasilnikov, P. Lotte, G. McKee, A. Malaquias, M. O'. Mullane, E. Rachlew, S.
} 
Tugarinov, and K.-D. Zastrow, 30th EPS Conf. Contr. Fus. Pl. Phys., St. Petersburg, 2003, ECA Vol. 27A, O-4.2D.

${ }^{3}$ A. Malaquias, A. Costley, A. Gorshkov, N. Hawkes, M. v. Hellermann, M. Kuldkepp, P. Lotte, E. Rachlew, S. Tugarinov, G. Vayakis, and C. Walker, 15th Topical Conference on High Temperature Plasma Diagnostics, San Diego, 19 April 2004.

${ }^{4}$ W. Mandl, R. Wolf, M. von Hellermann, and H. P. Summers, Plasma Phys. Controlled Fusion 35, 1373 (1993).

${ }^{5}$ M. von Hellermann, R. Jaspers, H. P. Summers, and K-D. Zastrow, Advanced Diagnostics for Magnetic and Inertial Fusion, edited by P. E. Stott, A. Wootton, G. Gorini, E. Sindoni, and D. Batani (Plenum, New York, 2001), p. 205.

${ }^{6}$ P. Lotte, M. Von Hellermann, A. Malaquias, R. Giannella, N. Hawkes, E. Rachlew, R. Jaspers, P. Nielsen, S. Tugarinov, and C. Walker, in Ref. 2, P1-183.

${ }^{7}$ A. Malaquias, M. von Hellermann, P. Lotte, S. Tugarinov, and V. Voitsenya, in Ref.2 .

${ }^{8}$ G. McKee, R. J. Fonck, B. Stratton, R. Budny, Z. Chang, and A. Ramsey,
Nucl. Fusion 37, 501 (1997).

${ }^{9}$ M. von Hellermann, W. G. F. Core, J. Frieling, L. D. Horton, R. W. T. König, W. Mandl, and H. P. Summers, Plasma Phys. Controlled Fusion 35, 799 (1993).

${ }^{10} \mathrm{~K}$. Vukolov and V. Voitsenya, Sixth ITPA Topical Group Meeting on ITER Diagnostics, Part I, Naka, Japan, February 2004.

${ }^{11}$ S. Tugarinov, A. Krasilnikov, V. Dokouka, R. Khayrutidinov, I. Beigman, I. Tolstikhina, L. Vainshtein, M. von Hellermann, and A. Malquias, Rev. Sci. Instrum. 74, 2075 (2003).

${ }^{12}$ N. C. Hawkes, A. Malaquias, P. Lotte, M. von Hellermann, M. Brix, R. Giannella, M. Kuldkepp, E. Rachlew, C. Negus, and E. Surrey, Fifth ITPA Topical Group Meeting on ITER Diagnostics, St. Petersburg, Russia, 16 July 2003.

${ }^{13}$ F. Levinton, R. Fonck, G. Gammel, R. Kaita, H. Kugel, E. Powell, and D. Roberts, Phys. Rev. Lett. 63, 2060 (1989).

${ }^{14}$ P. Wienhold, A. Litnovsky, V. Philipps, P. Oelhafen, G. De Temmerman, W. Schneider, and B. Emmoth. (unpublished).

${ }^{15} \mathrm{http}$ ://adas.phys.strath.ac.uk. 